\title{
MicroRNA-328 Inhibits Proliferation of Human Melanoma Cells by Targeting TGFB2
}

\author{
Jing-Rong Li ${ }^{1}$, Jian-Qin Wang ${ }^{1 *}$, Qing Gong ${ }^{2}$, Rui-Hua Fang ${ }^{1}$, Yun-Long Guo ${ }^{1}$
}

\begin{abstract}
Some microRNAs (miRNAs) have been shown to act as oncogenes or tumor suppressor genes in human melanomas. miR-328 is upregulated in blood cells of melanoma patients compared to in healthy controls. This suggests a role for miR-328 in melanoma that warrants investigation. In this study, we demonstrated miR-328 levels to be dramatically decreased in human melanoma cell lines. Moreover, forced expression of miR-328 inhibited proliferation and induced G1-phase arrest of the SK-MEL-1 melanoma cell line. We identified TGFB2 as a direct target gene for miR-328 using a fluorescent reporter assay and western blotting. Levels of TGFB2 were dramatically increased in human melanoma cell lines and were inversely correlated with the miR-328 expression level. Our findings provide new insights into the mechanisms of human melanoma development, indicating that miR-328 has therapeutic potential for this disease.
\end{abstract}

Keywords: miR-328 - TGFB2 - melanoma - proliferation

Asian Pac J Cancer Prev, 16 (4), 1575-1579

\section{Introduction}

Melanoma is a high-grade, poorly differentiated, malignant tumor that arises from melanocytes (pigmentproducing skin cells). Though an uncommon skin cancer, the prognosis of melanoma remains very poor (Glud et al., 2013; Gyrylova et al., 2014). And for most patients with melanoma, immunotherapy, chemotherapy, or small molecule inhibitors are not effective (Siegel et al., 2012). Improved understanding of the mechanisms behind the progression and metastasis of melanoma is essential to developing more effective therapies.

MicroRNAs (miRNAs) are small non-coding RNAs that regulate gene expression by translation repression or messenger RNA (mRNA) degradation (Leung et al., 2006; Nazari-Jahantigh et al., 2014). miRNAs have been shown to play a key role in the pathogenesis of many human diseases such as cancer (Phuah et al., 2014; Xu et al., 2014), and are involved in many biological processes, such as cell differentiation, tumorigenesis, cell death, proliferation, metastasis, and drug resistance (Ling et al., 2013; Di Leva et al., 2014; Phuah et al., 2014). Some miRNAs, such as microRNA-34 (miR-34) and let-7, have been shown to act as oncogenes or tumor suppressor genes in melanoma (Liu et al., 2014; Zhou et al., 2015). In a study by Leidinger and colleagues, microRNA-328 (miR-328) was found to be upregulated in blood cells of melanoma patients compared to blood cells of healthy controls (Leidinger et al., 2010). However, the function of miR-328 in melanoma is not clear. In human glioma, loss of miR-328 expression may stimulate advanced tumor progression and adverse outcome by promoting cellular proliferation and invasion, suggesting that miR-328 has a tumor suppressive role (Yuan et al., 2015). However, in another study, miR-328 promoted glioma cell invasion via SFRP1-dependent Wnt-signaling activation, indicating that miR-328 acts as an oncogene (Delic et al., 2014). Therefore, miR-328 might play a dual role in cancer, and its function in other cancers needs further study.

To investigate the role of miR-328 in melanoma, we examined the expression level of miR-328 in human epidermal melanocyte and human melanoma cell lines. We found that the expression level of miR-328 was decreased in the human melanoma cell lines A375 and SK-MEL-1. We then examined the effects of overexpressing miR328 in SK-MEL-1. In addition, we identified TGFB2 as a potential target of miR-328. Our results show that miR-328 may have a tumor suppressor role in the human melanoma cell line SK-MEL-1, acting through TGFB2.

\section{Materials and Methods}

\section{Cell lines and culture}

Human Epidermal Melanocytes (HEM) were purchased from Sciencell (USA) and cultured in Melanocyte Medium (Sciencell, USA) according to the manufacturer's instructions. Human melanoma cell lines SK-MEL-1 and A375 were obtained from American Type Culture Collection (Manassas, VA, USA). A375 and SKMEL-1 cells were maintained in RPMI-1640 medium or

${ }^{I}$ Department of Dermatology, Guangzhou First People's Hospital, ${ }^{2}$ Department of Biochemistry, Guangzhou Medical University, Guangzhou, Guangdong Province, China *For correspondence: gzsdyrmyynsyypfk@126.com 
DMEM medium respectively, supplemented with $10 \%$ fetal bovine serum, in a $37^{\circ} \mathrm{C}$ humidified atmosphere of $5 \% \mathrm{CO}_{2}$.

\section{miRNA mimic and TGFB2 transfection}

A miR-328 mimic and negative control (NC) were purchased from Jima Biotech (China). Cells were plated at $50 \%$ confluency and transfected with $200 \mathrm{nM}$ miR328 mimic or NC mimic using Lipofectamine 2000 (Invitrogen, USA) according to the manufacturer's protocol. Cells were harvested at 24 or $48 \mathrm{~h}$ after transfection for further analysis. The full-length TGFB2 open reading frame (NM_001135599) was cloned and inserted into expression plasmid pcDNA3.0. This plasmid was transfected using Lipofectamine 2000 according to the manufacturer's instructions.

RNA extraction and quantitative real-time PCR analysis

Total RNA was extracted from cultured SK-MEL-1 cells using Trizol reagent (Invitrogen, USA). miRNA was obtained, according to the manufacturer's instructions, using the miRNeasy FFPE Kit (Bioteke, Beijing, China). One microgram of RNA was reverse-transcribed using the First Strand cDNA Synthesis Kit (Promega, USA) with appropriate primers. miRNA or mRNA expression levels were quantitated using the SYBR Primescript RT-PCR Kit (TaKaRa, China) according to the manufacturer's instructions. Levels of gene transcripts were normalized to GAPDH. For miRNA analysis, the amount of miRNA was normalized to U6 using the comparative threshold cycle method. PCR was performed using the Mx3000P real-time PCR system (Agilent Stratagene, USA). Gene expression was measured in triplicate and quantified using the $2-\Delta \Delta \mathrm{CT}$ method normalized to a control.

\section{Cell viability assays}

SK-MEL-1 cell proliferation was monitored using the cell counting kit-8 (CCK-8) (Dojindo, Japan) according to the manufacturer's protocol. Twenty-four hours after transfection, cells were seeded at $2 \times 10^{3}$ per well in a 96well plate. Cell proliferation was measured at 24,48 , and $72 \mathrm{~h}$. Briefly, $10 \mu \mathrm{l}$ of WST- 8 was added to each well, and after $4 \mathrm{~h}$ incubation at $37^{\circ} \mathrm{C}$, the optical density (OD) value at $490 \mathrm{~nm}$ was measured using a Scan Reader.

\section{Flow cytometric analysis}

SK-MEL-1 cells were harvested $48 \mathrm{~h}$ after transfection. Cells were washed with PBS and fixed with $70 \%$ ethanol at $4{ }^{\circ} \mathrm{C}$. After overnight fixation, the cells were washed with PBS again and stained with propidium iodide (PI) using the Cell Cycle and Apoptosis Analysis Kit (Beyotime, China) for $30 \mathrm{~min}$. Cell cycle features were analyzed using the BD LSRII Flow Cytometer System with FACSDiva software (BD Biosciences, USA). Data analysis was performed using ModFit LT3.2 software (Verity Software House, USA).

\section{Western blotting}

SK-MEL-1 cells were lysed using RIPA buffer. Total protein concentration was determined with the BCA Protein Assay kit (Pierce, USA). Equal amounts of total protein were separated in $10 \%$ SDS polyacrylamide gels and transferred to polyvinylidenedifluoride membranes (PALL, USA). Membranes were blocked for $1 \mathrm{~h}$ at room temperature with $5 \%$ milk in TBS containing $0.05 \%$ Tween-20, incubated for $1 \mathrm{~h}$ with primary antibody (TGFB2, 1:1000, Abnova; GAPDH, 1:1000, Abcam), washed and incubated with secondary antibody, and visualized using Thermo Scientific Pierce ECL Plus.

\section{Vector construction and luciferase reporter assay}

The full-length wild-type 3' untranslated region (UTR) of TGF $\beta 2$ and mutant 3' UTR of TGFB2 were amplified and cloned into the psi-CHECK-2 vector (Promega, USA). All plasmids were confirmed by DNA sequencing. SKMEL-1 cells plated on 24-well plates were co-transfected with $100 \mathrm{ng}$ plasmid and $200 \mathrm{nmol} / \mathrm{L}$ of miR-328 mimic or negative control. Cell lysates were harvested $48 \mathrm{~h}$ after transfection and then firefly and Renilla luciferase activities were measured by the Dual-Luciferase Reporter Assay System (Promega, USA) according to the manufacturer's instructions. Three independent experiments were performed.

\section{Statistical analysis}

All statistical analysis was performed by SPSS 19.0 software (SPSS Inc., USA). All data were expressed as the mean \pm standard deviation (SD). The differences between groups were assessed using Student's t-test. Differences between groups were considered significant at $\mathrm{P}<0.05$.

\section{Results}

miR-328 is down-regulated in human melanoma cell lines

To investigate whether miR-328 is involved in tumorigenesis in human melanoma cells, we first examined the expression level of miR-328 in human melanoma cells (SK-MEL-1 and A375) and in human epidermal melanocytes (HEM). As shown in Figure 1, the expression level of miR-328 was significantly lower in melanoma cells (SK-MEL-1 and A375) than in HEM cells. The expression level of miR-328 was lowest in SK-MEL-1 cells, so SK-MEL-1 cells were chosen for the following assays.

\section{A}

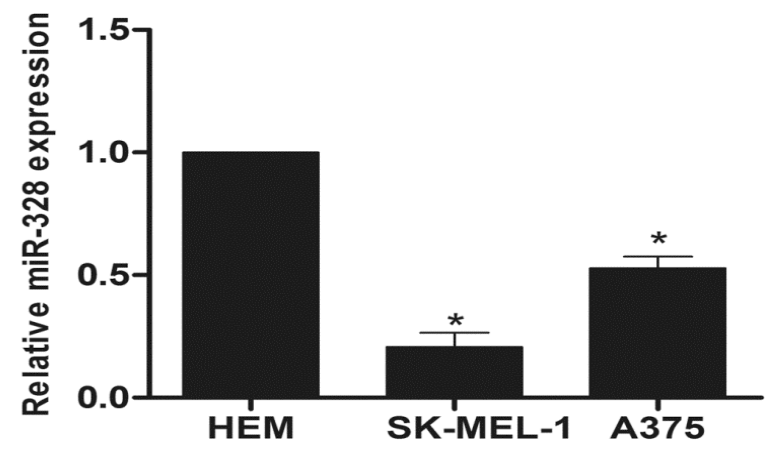

Figure 1. miR-328 Expression Levels in Human Epidermal Melanocyte (HEM) and Human Melanoma Cell Lines SK-MEL-1 and A375. Data are expressed as mean \pm SD. $* p<0.05$ 
miR-328 inhibits SK-MEL-1 cell growth

As miR-328 expression levels were significantly lower in melanoma cells, we hypothesized that miR-328 functions as an oncosuppressor. To test this, we transfected SK-MEL-1 cells with a miR-328 mimic or a NC mimic, and miR-328 expression levels were successfully up-regulated (Figure 2A). miR-328 overexpression dramatically inhibited SK-MEL-1 cell proliferation compared with NC transfection (Figure 2B). After 48 h of miR-328 transfection, we observed a significant inhibition of SK-MEL-1 cell growth that persisted and became more significant at $72 \mathrm{~h}$ (Figure $2 \mathrm{~B}$ ).

\section{miR-328 induces G1-phase arrest}

To uncover the underlying mechanisms of cell proliferation suppression by miR-328, we used flow cytometry to observe the distribution of cell cycle stages after transfection of a miR-328 mimic or NC mimic.
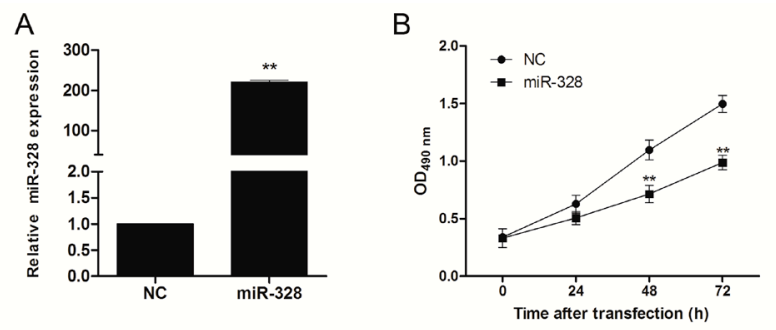

Figure 2. Effect of miR-328 Expression on SK-MEL-1 Cell Proliferation. Expression of miR-328 in SK-MEL-1 cells transfected with a miR-328 mimic or negative control (NC) was quantified by real-time PCR. B: Optical density (OD) at $490 \mathrm{~nm}$ was used to assess cell proliferation at $0,24,48$, and $72 \mathrm{~h}$ after miR-328 mimic or NC transfection. ${ }^{* *} p<0.01$

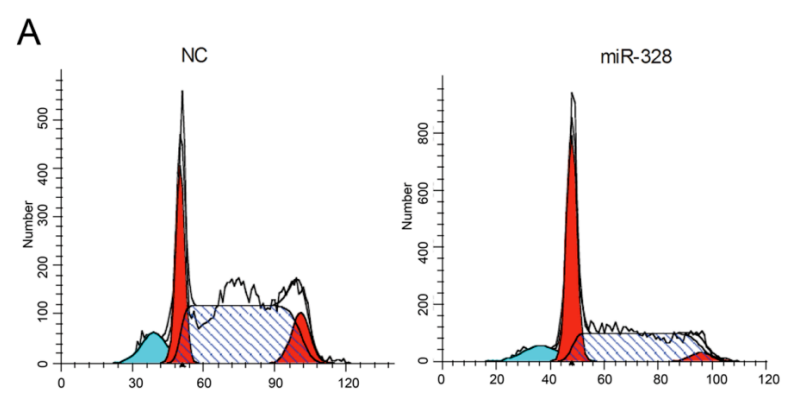

B

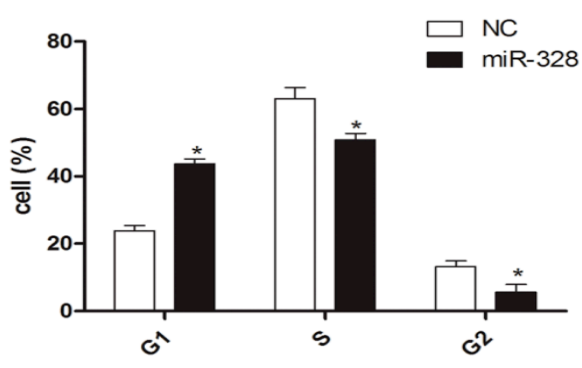

Figure 3. Effect of miR-328 Overexpression on the Cell Cycle. A: Representative image of SK-MEL-1 cell cycle stages measured by flow cytometry $24 \mathrm{~h}$ after transfection with a miR-328 mimic or negative control (NC). B: Proportions of cells in each cell cycle phase following transfection with a miR-328 mimic or NC. Data are presented as mean \pm SD. $* p<0.05$
Overexpression of miR-328 induced a significant G1phase arrest in SK-MEL-1 (Figure 3), and the percentage of cells in S and G2 phase decreased significantly. These results suggest that miR-328 is a suppressor of cell proliferation in the human melanoma cell line SK-MEL-1.

\section{TGFB2 is a direct target of miR-328}

To understand the mechanisms by which miR-328 inhibited tumor cell proliferation, we used several computational methods to help identify miR-328 targets in humans. We found that miR-328 has a binding site in the 3'UTR of TGFB2 (Figure 4A). We also examined the expression level of TGFB2 in SK-MEL-1 and A375 human melanoma cell lines compared to HEM cells. Protein levels of TGFB2 in SK-MEL-1 and A375 cells were dramatically increased compared to HEM cells (Figure 4B). To test whether TGFB2 is a direct target of miR-328, we carried out luciferase reporter assays. As expected, miR-328 inhibited luciferase activity downstream of the wild-type 3'UTR of TGFB2. In contrast, miR-328 had no effect on luciferase activity downstream of a mutant 3'UTR of TGFB2 (Figure 4C). We then examined the effect of miR-328 overexpression on TGFB2 mRNA and protein levels. miR-328 overexpression did not cause degradation of TGFB2 mRNA (Figure 4D). However, a clear reduction in the level of endogenous TGFB2 protein was observed (Figure 4E).

Overexpression of TGFB 2 reversed the effect of $m i R-328$ on cell proliferation

To further examine whether miR-328 affects SK-

A $\begin{array}{ccccc}\text { 3' } & \text { UTR Mutant } & \text { 5' } & \text { GUUCUGCCAACGCCACAGGAAA } \\ \text { 3' } & \text { UTR Wild } & \text { 5' } & \text { GUUCUGCCAACGCCAGGGCCAA } \\ & \text { I | | | | | | } \\ & \text { miR-328 } & \text { 3' } & & \text { UGCCUUCCCGUCUCUCCCGGUC }\end{array}$

B

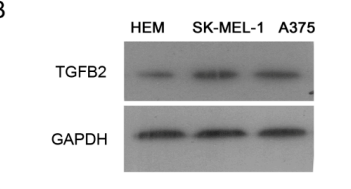

D
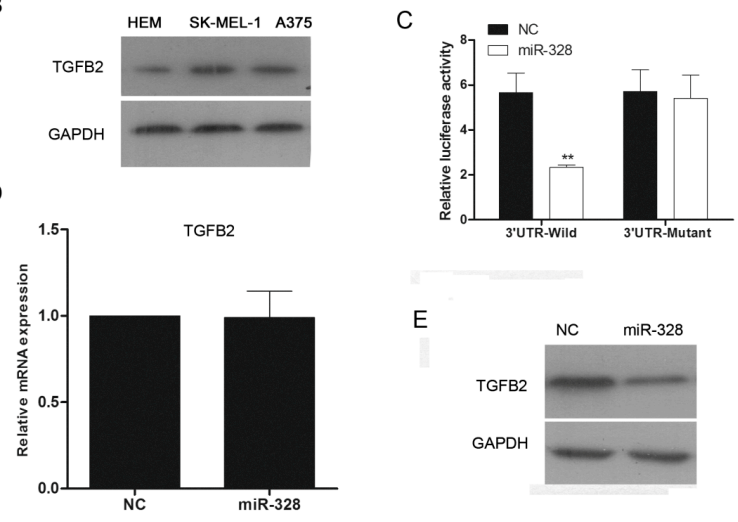

E

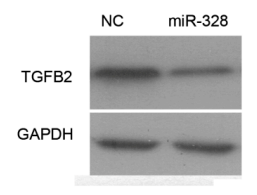

Figure 4. TGFB2 is a Direct Target of miR-328. A: Predicted duplex formation between the wild-type or mutant TGFB2 3'UTR and miR-328. B: TGFB2 protein expression levels in Human Epidermal Melanocyte (HEM) and human melanoma cell lines SK-MEL-1 and A375. C: Luciferase activity of wild-type ( $3^{\prime}$ UTR-Wild) or mutant (3`UTR-Mutant) TGFB2 3'UTR reporters in SK-MEL-1 cells transfected with a miR-328 mimic or negative control (NC). D: Real-time reverse transcription PCR (RT-PCR) data for TGFB2 mRNA in SKMEL-1 cells transfected with a miR-328 mimic or NC. Data were normalized to GAPDH mRNA. E: Western blot of TGFB2 in SK-MEL-1 cells transfected with a miR-328 mimic or NC. Data are expressed as mean $\pm \mathrm{SD}, * * p<0.05$ 
Jing-Rong Li et al

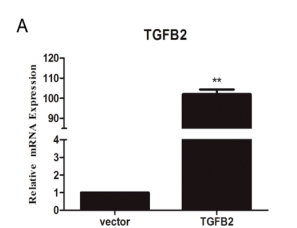

B
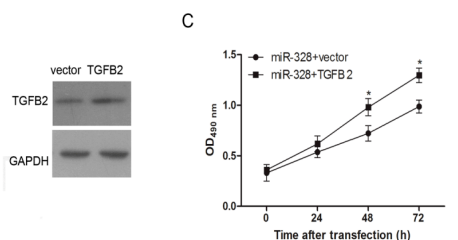

D

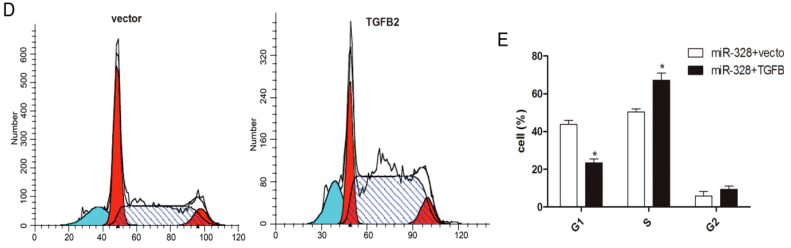

Figure 5. Effect of TGFB2 Overexpression on cell Growth and the Cell Cycle after miR-328 Transfection. A: TGFB2 mRNA expression $48 \mathrm{~h}$ after TGFB2 transfection. B: TGFB2 protein expression $48 \mathrm{~h}$ after TGFB2 transfection. C: TGFB2 overexpression reversed the effect of miR-328 on cell proliferation. D: Representative image of SK-MEL-1 cell cycle stages measured by flow cytometry $24 \mathrm{~h}$ after transfection of a miR-328 mimic and TGFB2 overexpression vector, or miR-328 mimic and empty vector. E: Proportions of cells per cell cycle phase $24 \mathrm{~h}$ after transfection of a miR-328 mimic and TGFB2 overexpression vector, or miR-328 mimic and empty vector. Data are expressed as mean $\pm \mathrm{SD},{ }^{*} p<0.05$

MEL-1 cell proliferation through TGFB2, a TGFB2 overexpression vector was constructed and transfected into SK-MEL-1 cells. As shown in Figure 5A and 5B, TGFB2 mRNA and protein levels increased after transfection of the vector. SK-MEL-1 cell proliferation was increased following transfection of both miR-328 and TGFB2 compared to miR-328 and an empty vector (Figure 5C). Forced expression of TGFB2 also rescued miR-328induced G1-phase arrest (Figure 5D and 5E). These results suggest that miR-328 affects SK-MEL-1 cell proliferation through regulation of its target TGFB2.

\section{Discussion}

In the present study, we have shown that miR-328 expression is significantly decreased in human melanoma cells compared with human epidermal melanocytes. Moreover, we showed that miR-328 inhibited proliferation of the human melanoma cell line SK-MEL-1 and induced G1-phase arrest in vitro. TGFB2 is negatively regulated by miR-328 at the post-transcriptional level, via a specific target site within the 3'UTR. Furthermore, expression levels of TGFB2 were significantly increased in human melanoma cells compared to human epidermal melanocytes, and were inversely associated with the expression of miR-328. The identification of miR-328 as an important regulator of human melanoma cell proliferation emphasizes an essential role for this miRNA in mediating human melanoma oncogenesis.

In blood cells of melanoma patients, miR-328 was found to be upregulated compared to blood cells of healthy controls (Leidinger et al., 2010). So it would be reasonable to predict that the expression level of miR328 would be increased in human melanoma cell lines compared to human epidermal melanocytes. However, our results showed that miR-328 is downregulated in human melanoma cell lines. These results indicate that miR-328 might play different roles in different cells in the initiation and progression of human melanoma.

Some studies have shown that miR-328 can act as an oncogene or tumor suppressor gene in cancer (Wu et al., 2012; Delic et al., 2014; Yang et al., 2014; Yuan et al., 2015). This may be due to its ability to regulate genes involved in diverse aspects of proliferation and metastasis. However, the expression and biological function of miR328 in melanoma remains unclear. Our results have shown that the expression of miR-328 in human melanoma cell lines was significantly lower than that in human epidermal melanocytes. In addition, overexpression of miR-328 significantly inhibited cell proliferation. In summary, our results suggest that miR-328 contributes to the prevention of human melanoma, acting as potential tumor suppressor.

In this study, TGFB2 was identified as a direct target of miR-328 in SK-MEL-1 cells. Complementary sequence of miR-328 was identified in the 3'UTR of TGFB2 mRNA, and overexpression of miR-328 led to a significant reduction in TGFB2 protein. miR-328 overexpression suppressed TGFB2 3'UTR luciferase reporter activity and this effect was abolished by mutation of the miR-328 seed binding site. In addition, overexpression of TGFB2 reversed the effect of miR-328 on cell proliferation. These results indicate that miR-328 may function as a tumor suppressor in human melanoma, mediated partly through repressing TGFB2 expression.

TGFB2 is a member of the transforming growth factor $\beta$ (TGFB) family of cytokines. These are multifunctional peptides that regulate proliferation, differentiation, adhesion, migration, and other functions in many cell types, by signal transduction through combinations of transmembrane type I and type II receptors and their downstream effectors, the SMAD proteins (Yao et al., 2008; Sethi et al., 2011; Joko et al., 2013; Scola et al., 2014). The expression level of TGFB2 is significantly increased in B-lymphoma and glioblastoma (He et al., 2014; Wotton, 2014). Our results showed that the expression of TGFB2 was increased in human melanoma cell lines A375 and SK-MEL-1. Moreover, the expression of TGFB2 was negatively correlated with miR-328 levels. So, upregulation of miR-328 in SK-MEL-1 cells may inhibit the expression of TGFB2, inhibiting proliferation of the cancer.

In conclusion, the current study provides novel evidence that miR-328 restrains human melanoma cell proliferation partly through repression of TGFB2. Our findings suggest that miRNA-328 might be a potential candidate for the diagnosis and treatment of human melanoma in future.

\section{References}

Delic S, Lottmann N, Stelzl A, Liesenberg F, Wolter M, Gotze $\mathrm{S}$, et al. (2014). MiR-328 promotes glioma cell invasion via SFRP1-dependent Wnt-signaling activation. Neuro Oncol, 16, 179-90.

Di Leva G, Garofalo M, Croce CM (2014). MicroRNAs in cancer. Annu Rev Pathol, 9, 287-314.

Glud M, Gniadecki R (2013). MicroRNAs in the pathogenesis of malignant melanoma. J Eur Acad Dermatol Venereol, 
$27,142-50$

Gyrylova SN, Aksenenko MB, Gavrilyuk DV, et al (2014). Melanoma incidence mortality rates and clinico-pathological types in the Siberian area of the Russian Federation. Asian Pac J Cancer Prev, 15, 2201-4.

$\mathrm{He} \mathrm{DM}, \mathrm{Wu} \mathrm{H}, \mathrm{Wu} \mathrm{XL}$, et al (2014). The gene expression patterns of BMPR2, EP300, TGFbeta2, and TNFAIP3 in B-lymphoma cells. Cancer Biol Med, 11, 202-07.

Joko T, Shiraishi A, Akune Y, et al. (2013). Involvement of P38MAPK in human corneal endothelial cell migration induced by TGF-beta (2). Exp Eye Res, 108, 23-32.

Leidinger P, Keller A, Borries A, et al (2010). High-throughput miRNA profiling of human melanoma blood samples. $B M C$ Cancer, 10, 262.

Leung AK, Sharp PA (2006). Function and localization of microRNAs in mammalian cells. Cold Spring Harb Symp Quant Biol, 71, 29-38.

Ling H, Fabbri M, Calin GA (2013). MicroRNAs and other noncoding RNAs as targets for anticancer drug development. Nat Rev Drug Discov, 12, 847-65.

Liu R, Xie H, Luo C, et al. (2014). Identification of FLOT2 as a novel target for microRNA-34a in melanoma. J Cancer Res Clin Oncol, 18, 18.

Nazari-Jahantigh M, Egea V, Schober A, Weber C (2014). MicroRNA-specific regulatory mechanisms in atherosclerosis. J Mol Cell Cardiol, S0022-2828, 342-3.

Phuah NH, Nagoor NH (2014). Regulation of microRNAs by natural agents: new strategies in cancer therapies. Biomed Res Int, 804510, 1.

Scola L, Di Maggio FM, Vaccarino L, et al (2014). Role of TGFbeta pathway polymorphisms in sporadic thoracic aortic aneurysm: rs900 TGF-beta2 is a marker of differential gender susceptibility. Mediators Inflamm, 165758, 24.

Sethi A, Jain A, Zode GS, Wordinger RJ, Clark AF (2011). Role of TGFbeta/Smad signaling in gremlin induction of human trabecular meshwork extracellular matrix proteins. Invest Ophthalmol Vis Sci, 52, 5251-59.

Siegel R, DeSantis C, Virgo K, et al (2012). Cancer treatment and survivorship statistics, 2012. CA Cancer J Clin, 62, 220-41.

Wotton D (2014). A CREB1-TGFbeta2 self-sustaining loop in glioblastoma. Cancer Discov, 4, 1123-25.

Wu Z, Sun L, Wang H, et al (2012). MiR-328 expression is decreased in high-grade gliomas and is associated with worse survival in primary glioblastoma. PLoS One, 7, 12.

$\mathrm{Xu}$ XD, Wu XH, Fan YR, (2014). Exosome-derived microRNA$29 \mathrm{c}$ induces apoptosis of BIU-87 cells by down regulating BCL-2 and MCL-1. Asian Pac J Cancer Prev, 15, 3471-6.

Yang SF, Lee WJ, Tan P, et al (2014). Upregulation of miR328 and inhibition of CREB-DNA-binding activity are critical for resveratrol-mediated suppression of matrix metalloproteinase- 2 and subsequent metastatic ability in human osteosarcomas. Oncotarget, 30, 30.

Yao K, Ye PP, Tan J, Tang XJ, Shen Tu XC (2008). Involvement of PI3K/Akt pathway in TGF-beta2-mediated epithelial mesenchymal transition in human lens epithelial cells. Ophthalmic Res, 40, 69-76.

Yuan J, Zheng Z, Zheng Y, et al (2015). microRNA-328 is a favorable prognostic marker in human glioma via suppressing invasive and proliferative phenotypes of malignant cells. Int J Neurosci, 6, 1-22.

Zhou Y, Zhang L, Fan J, et al (2015). Let-7b overexpression leads to increased radiosensitivity of uveal melanoma cells. Melanoma Res, 13, 13. 